\title{
Comunicación y cooperación para el desarrollo
}

\begin{abstract}
Alfons Martinell Sempere, Universidad de Girona - alfons.martinell@udg.edu
Gemma Carbó Ribugent, Universidad de Girona - gemma.carbo@udg.edu
\end{abstract}

\begin{abstract}
The article describes the process of incorporating communication in culture and development strategies in international cooperation based on the analysis of information. Likewise presents the formulation of a cultural approach to development where communication plays an important role. From the analysis of documents and research, proposes greater integration of communication -culture cooperation axis.
\end{abstract}

Keywords

Communication, culture, cooperation, diversity, development.

Resumen

El artículo relata el proceso de incorporar la comunicación en las estrategias de cultura y desarrollo en la cooperación internacional a partir del análisis de la información. De la misma forma presenta la formulación de un enfoque cultural para el desarrollo donde la comunicación tiene un papel importante. A partir del análisis de documentos e investigaciones propone una mayor integración del eje comunicación - cooperación -cultura.

\section{Palabras clave}

Comunicación, cultura, cooperación, diversidad, desarrollo.

Sumario

1. Antecedentes y marco de referencia. 2. Comunicación, cooperación y cultura. 3. 3. La comunicación en el enfoque cultural en la cooperación al desarrollo. 4. Conclusiones. 5. Bibliografía. 


\section{Antecedentes y marco de referencia}

Las relaciones entre comunicación y cooperación al desarrollo tienen una larga tradición tanto en el entorno de los organismos internacionales como en la práctica de las agencias nacionales y las ONG's. El Programa para el desarrollo de la comunicación de UNESCO con más de 30 años de experiencia y su continuidad es un ejemplo a tener en cuenta aunque en sus objetivos se orientan más a la libertad de expresión y los medios de comunicación, con poca relación con el sector cultura. Por otro lado los cambios de la comunicación en las sociedades más globalizadas replantean algunos de estos postulados y se perfila una nueva línea o enfoque más amplio en respuesta a nuevas necesidades, como se ha expresado en diferentes foros.

«la comunicación para el desarrollo es un proceso social basado en el diálogo, usando un amplio abanico de instrumentos y de métodos. Se refiere a la búsqueda del cambio a diferentes niveles, que incluyen escuchar, construir confianza, compartir conocimiento y habilidades, desarrollar políticas, debatir y aprender para lograr cambios sostenibles y significativos. No se trata de relaciones públicas ni de comunicación corporativa»

Dentro de los múltiples trabajos realizados destacamos por la proximidad al contenido de este artículo los trabajos de (Bustamante, 2007) (Martínez-Gómez,- Lubetkin, 2011) (De Bustos, 2006) donde se incide en la necesidad de una mayor articulación entre comunicación, cooperación y cultura

Las conclusiones de la investigación realizada por la Universidad de Girona nos permite aportar las lecciones aprendidas en este campo fruto de un proceso de gestión de conocimiento a partir de la experiencia práctica en 18 Programas conjuntos de la Ventana de Cultura y Desarrollo en diferentes continentes. Los resultados evidencian la necesidad de incorporar más y mejor la comunicación en sus acciones. (FODM \& Universidad de Girona, 2013)

En este artículo se presentan algunas reflexiones sobre los procesos orientados a incorporar la comunicación como un elemento constitutivo en las estrategias sectoriales de cultura y desarrollo a partir de la experiencias innovadoras que se iniciaron en la Cooperación Española y en el FODM que inciden en la configuración de una marco de referencia específico para situar la cultura y la comunicación de forma más concluyente en las estrategias de desarrollo en la cooperación internacional.

\section{Comunicación, cooperación y cultura}

La incorporación de la cultura en los planes directores de la cooperación española se define en el II Plan Director (MAEC ,2005) con la voluntad de incorporar una mayor especificidad del sector cultural como un eje fundamental para las políticas de desarrollo, que después alcanzarían otros fines de acuerdo con nuevos lineamientos en los organismos multilaterales.

Esta política se concretó en la Estrategia de Cultura y Desarrollo de la Cooperación Española que dentro de sus diferentes objetivos incorpora una línea específica en el campo de la Comunicación (MAEC, 2007;25) con la finalidad de:

"'Potenciar procesos autónomos propios en el ámbito comunicativo, de acuerdo con la realidad de cada contexto.

Contribuir a la inserción de los diferentes sectores que componen la comunidad en las distintas dimensiones de las dinámicas y procesos comunicativos.

Trabajar en la formación de públicos, la mejora de la distribución y el acceso a los medios. Generar nuevas industrias culturales y estimular procesos que evolucionen de la cooperación a la coproducción."”

Estos avances en la conformación de un papel más relevante de la cultura en las políticas de cooperación al desarrollo también van definiendo una mayor relación entre cultura - comunicación y desarrollo. Donde la consideración de los medios, espacios y redes de comunicación se convierten en unos elementos imprescindibles para los fines estratégicos de la cooperación. La incorporación de la cultura como un sector para el desarrollo concibe la cooperación como el conjunto de procesos de comunicación simétricos, horizontales y dialógicos entre los actores involucrados que facilitan la acción en común. Lo cual significa la superación de formas de dependencia entre las partes y la instauración de un sistema de intercambio permanente para conseguir los fines del acuerdo de cooperación.

Entre diferentes aportaciones citadas destaca el trabajo realizado en la investigación aplicada (FODM \& Universidad de Girona ,2013), Sus resultados son aplicaciones prácticas que permiten tener en cuenta algunos elementos para una mejor integración de la comunicación en los procesos de cooperación al desarrollo en la dimensión cultural:

- $\quad$ Fomentar la comunicación horizontal y participativa para facilitar los procesos de empoderamiento y generación de capacidades de expresión y comunicación de las partes en cooperación.

- $\quad$ Adecuar la acción comunicativa a la realidad de cada contexto. Por medio de procesos de conocimiento de los espacios, tiempos, lenguajes y redes culturales de cada contexto.

- Identificación de las capacidades necesarias para una comunicación eficaz en proyectos de cooperación al desarrollo.

- Conocimiento de la vida cultural, las artes, formas de vida y las expresiones culturales como base de una comunicación eficaz y participativa

- $\quad$ Considerar la comunicación como un proceso permanente y que deben mantenerse en los diferentes momentos del ciclo del proyecto de cooperación

Incorporar la comunicación en los procesos de cooperación, y más concretamente en la dinámica cultural, reclama una mayor vinculación con los contextos próximos, fomentando una participación más activa de la ciudadanía frente al tradicional función de los estamentos gubernamentales con el fin de crear un espacio de comunicación estratégica entre las partes. Como dice (Martin Barbero, 2011) «el modelo anterior de comunicación vertical unidireccional ha caducado con el tiempo, dejando espacio a la comunicación de tipo horizontal o comunicación red, que significa conectividad e interacción, transformando la mecánica forma de la conexión a distancia por la electrónica de la interfaz y la proximidad». Esto supone un cambio profundo en la comunicación de la cultura entre los agentes 0 actores que intervienen como en las relaciones con la ciudadanía, usuarios, consumidores y públicos. Para ello se requerirá disponer de nuevas capacidades en las poblaciones, en una perspectiva de desarrollo humano, para asumir un nuevo campo de relaciones entre cooperación al desarrollo y la comunicación.

En el marco de la cooperación al desarrollo se considera imprescindible situar la comunicación como un factor relevante que incide en la reducción de las brechas con las poblaciones más vulnerables y fomenta la participación y apropiación de la ciudadanía para dirigir sus propias transformaciones para el desarrollo. Los procesos comunicativos refuerzan las capacidades de los individuos y la comunidad en su construcción de ciudadanía, auto reconocimiento e identidad cultural. 
En la sociedad de la información, cada vez más globalizada, el acceso a la "conectabilidad" y la posibilidad de actuar a través de los diferentes medios, sistemas y redes se convierte en un elemento imprescindible para el buen funcionamiento de la cooperación como para conseguir los resultados de desarrollo que se proponen. El dominio de capacidades comunicativas amplias es imprescindible para los procesos de desarrollo para evitar desigualdades y exclusiones de una parte de las poblaciones más excluidas. Por otro lado la estrategia de comunicación en los proyectos y planes de cooperación al desarrollo adquieren más importancia, tanto por la necesidad de emitir una imagen más fidedigna a su función en la comunidad internacional, como en la propia adquisición de capacidades comunicativas de los destinatarios o sujetos activos de estos planes, que por medio de la utilización de estrategias comunicativas pueden apropiarse del medio y pueden emitir a partir de su realidad, cultura y visión del mundo.

La incorporación de estrategias comunicativas innovadoras y participativas tiene más sentido cuando existe un compromiso de la comunidad internacional en la protección de la diversidad en las expresiones culturales que se articula en diferentes programas y fondos. Para fomentar los valores que defiende la diversidad cultural requiere de una alianza con medios de comunicación y la participación de amplios sectores de la sociedad en los canales y redes sociales que permiten la circulación de visiones culturales más cercanas a los propios actores, convirtiéndose en emisores de su propia vida cultural.

De la misma forma las instituciones gubernamentales han de incidir en estos aspectos de la vida cultural que requiere la implicación de los agentes culturales, la industria creativa y los medios de comunicación tradicional en el reconocimiento de la diversidad cultural, saberes plurales y el dialogo intercultural. La interacción de estos diferentes actores puede fomentar los procesos de reconocimiento cultural, fomentar la creatividad y la diversidad de expresiones culturales frente a la tendencia a una homogenización de los contenidos culturales. Por estas razones las experiencias analizadas en el campo de la cooperación al desarrollo aportan conocimiento relevante y algunas conclusiones o recomendaciones.

La cooperación al desarrollo ha de considerar la comunicación desde tres ámbitos:

- La comunicación como derecho y capacidad humana fundamental. El derecho a la comunicación y el fomento de la comunicación como capacidad básica pare el desarrollo permite los procesos de apropiación y participación

- $\quad$ La comunicación como un sistema de medios, estrategias y técnicas que facilitan la trasferencia, visibilidad de las acciones de cooperación al desarrollo

- La comunicación como un sector económico estratégico en las sociedades contemporáneas a partir de las industrias creativas, los medios de comunicación y las nuevas actividades que pueden realizarse por medio de internet y los instrumentos de información generalizada.

Los postulados de la Estrategia de Cultura y Desarrollo (MAEC, 2007) han permitido avanzar en la implementación de programas y acciones que han reforzado estos planteamientos y han permitido progresar en la formulación de sistemas y metodologías más concretas y adaptadas a este campo de la cooperación. El conjunto de estos saberes y conocimientos han permitido evolucionar desde una propuesta inicial al planteamiento de un Enfoque Cultural para el Desarrollo que va integrando los aprendizajes de las experiencias y formulando un campo más definido y técnico. A partir de estos antecedentes se propone que la comunicación esté presente en este enfoque de acuerdo con algunos de los elementos que a continuación se presentan.

\section{La comunicación en el enfoque cultural en la cooperación al desarrollo}

Desde la Cumbre del Milenio en el año 2000 y el establecimiento de los Objetivos del Milenio se ha manifestado una gran preocupación por encontrar una mayor eficacia a la reducción de la pobreza, al desarrollo sostenible en las políticas de cooperación internacional al desarrollo. Estos procesos han ido evolucionando el concepto de desarrollo abriéndose a diferentes interpretaciones por los diferentes actores, los cuales bajo el derecho a participar en su propio desarrollo han cuestionado ciertas visiones tradicionales procedentes de posiciones dominantes en el contexto internacional (De Bustos,2006; Martinell, 2010). A partir de estas acciones se ha configurado una comunidad de conocimiento especializada que considera que la contribución de la cultura al desarrollo es una variable imprescindible. Por esta razón se ha de avanzar hacia una mayor definición de un marco de referencia específico que definimos como un Enfoque Cultural para el Desarrollo. Dentro de este plnteamiento se incorpora de forma muy explícita la comunicación de acuerdo con estos postulados:

Se requiere replantear las relaciones entre comunicación, cultura y cooperación al desarrollo que están muy condicionadas por las posiciones dominantes de ciertos grupos, como medios de comunicación, la comunicación gubernamental y la institucionalidad que alteran el verdadero rol que la comunicación puede ejercer en el desarrollo humano y la cooperación cultural

Aceptar que la comunicación supone reconocer las diferentes formas de ver el mundo en diversidad en la circulación de la información, lo medios tradicionales y las nuevas formas de comunicación.

La comunicación cultural, en contextos de cooperación, se entiende como un amplio abanico de procesos y dinámicas que sobrepasan las limitadas perspectivas de estrategias de comunicación centradas en servicios de prensa, marketing, etc... de planes, proyectos u organizaciones.

La diversidad cultural, la creatividad, la expresividad y las formas de vida cultural son elementos estratégicos para el desarrollo humano, el bienestar y para el fomento del sector cultural y las industrias creativas. Estas estrategias requieren de una participación activa de las personas, grupos, comunidades en un marco de relación que reconozca los derechos fundamentales, la libertad cultural y la libertad de información.

El uso de todas las posibilidades que ofrece la sociedad de la información, internet y sus nuevos sistemas es imprescindible para aprovechar todo su potencial y favorecer la participación en la vida cultural de toda la población. En estas dimensiones adquieren una gran importancia los accesos a la cultura y a la conexión como un servicio público básico para poder usar los canales de comunicación en igualdad de oportunidades.

Para una mayor eficacia de la cooperación es necesario tener en cuenta la realidad y los valores de la población local en los procesos comunicativos. Reconociendo los actores involucrados en los proceso de comunicación para procurar su implicación, apropiación y sostenibilidad.

La comunicación ha de actuar de modo que evite la reproducción de estigmas y subvaloraciones culturales por su condición étnica, económica, política, etc.. respetando los derechos humanos y la diversidad cultural como principios de la comunicación en el marco de la cooperación al desarrollo.

\section{Conclusiones}


Los trabajos realizados desde la aprobación de la Estrategia de Cultura y Desarrollo (MAEC, 2007) y su implementación en diferentes proyectos, planes y fondos de la cooperación al desarrollo internacional bajo la dimensión cultural han evidenciado su importancia y consideración de acuerdo con los aportes de este sector a la agenda post-2015 de la comunidad internacional.

En el estudio y análisis de las intervenciones en este campo se ha observado una mayor implicación y sinergia entre cultura y comunicación de acuerdo con las nuevas realidades de estos campos en las sociedades contemporáneas. Incorporando esta relación en el Enfoque Cultural para el Desarrollo como una herramienta específica de formulación y seguimiento para los actores de estas actuaciones. Por otro lado las procesos de gestión del conocimiento, evaluaciones y prospectivas plantean la necesidad de una investigación más transversal de los diferentes sectores para la cooperación al desarrollo. En este sentido los estudios culturales y los estudios de comunicación pueden encontrar un campo de interdisciplinariedad muy importante y conveniente. Los análisis de las experiencias prácticas evidencian la necesidad de disponer de más conocimiento aplicado para asumir la complejidad actual de las relaciones entre desarrollo y cultura, donde la comunicación ha de incorporarse a partir de los planteamientos que algunos autores ya han presentado en este campo. En este sentido, investigadores y gestores están llamados a la cooperación para mejorar la eficacia y responder a los retos de la nueva agenda post 2015 del desarrollo a nivel internacional.

\section{Bibliografía}

Asamblea General de las Naciones Unidas. Resolución 50/103. La comunicación para los programas de desarrollo en el sistema de las Naciones Unidas. 23 de febrero de 1996.

Bustamante,E. (ed.) (2007). La cooperación cultura-comunicación en Iberoamérica. Colección Cultura y Desarrollo. Agencia Española de Cooperación Internacional.

FAO.(2006) Consenso de Roma, documento final del Congreso Mundial sobre Comunicación para el Desarrollo (CMCD). Roma. http://www.fao.org/nr/com/abst/com_081201_es.htm

FODM \& Universidad de Girona (2013): Learning from practical experiences. Culture \& Development. Accesible en http://www.aplcultural.com/ (Ver documento y aplicación: Procesos de Comunicación en proyectos bajo el enfoque cultural para el desarrollo.

MAEC (2005). II Plan director de la cooperación española. 2005-2008

MAEC (2007). Estrategia cultura y Desarrollo de Cooperación Española. Madrid

MAEC (2009). III Plan director de la cooperación española. 2009-2012

Martín Barbero, J. (2011) «Las relaciones entre comunicación para el desarrollo». Cátedra UNESCO de Políticas Culturales y Cooperación de la Universidad de Girona.

Martinell, a coord. (2010): Cultura y Desarrollo. Un compromiso para la libertad y el bienestar, Madrid, Siglo XXI.

Martínez-Gómez, Raquel y Lubetkin, Mario. (2011)Políticas, redes y tecnologías en la comunicación para el desarrollo. Zamora, Comunicación Social Ediciones y Publicaciones.

NNUU (2011): Comunicación para el Desarrollo. Fortaleciendo la eficacia de las Naciones Unidas. New York. PNUD.

UNESCO. (2005) Informe mundial de la UNESCO. Hacia las sociedades del conocimiento. 2005:. unesdoc.unesco.org/images/0014/001419/141908s.pdf

UNESCO (2005): Convención sobre la Protección y Promoción de la Diversidad de las Expresiones Culturales.

\section{Cómo citar este artículo en bibliografías - How to cite this article in bibliographies / references:}

MARTINELL-SEMPERE, A.; CARBÓ-RIBUGENT, G. (2015): “Comunicación y cooperación para el desarrollo". En Revista de la Asociación Española de Investigación de la Comunicación, vol. 2, número 4, pp. 8-11. 